\title{
Vagus Nerve Injury during Continuous Intraoperative Neuromonitoring (cIONM) for Thyroid Surgery: Assessment of Severity
}

\author{
M.Rohaizak ${ }^{a}$, AR Nor Faezan ${ }^{b}$, o Suraya ${ }^{b}$, AS Shahrunniza $^{a}$ \\ ${ }^{a}$ Endocrine and Breast Surgical Unit, Faculty of Medicine, Universiti Kebangsaan Malaysia, Cheras, Kuala Lumpur, Malaysia \\ ${ }^{b}$ Department of Surgery, Faculty of Medicine, Universiti Teknologi MARA (UiTM), Selayang Campus, Batu Caves, Selangor Darul Ehsan.
}

Keywords

nerve monitor, vagus nerve, recurrent laryngeal,

vocal cord palsy

Corresponding Author

Prof Dr Rohaizak Muhammad

Faculty of Medicine

Universiti Kebangsaan Malaysia,

Jalan Yaacob Latif, Bandar Tun Razak,

56000, Cheras, Kuala Lumpur, Malaysia

Malaysia.

Email: rohaizak@hotmail.com

Received: 5 October 2020; Accepted: 13

February 2021

Doi: https://doi.org/10.31436/imjm.v20i3

\section{ABSTRACT}

INTRODUCTION: Vocal cord palsy is one of the major concerns in thyroid surgery especially among professional voice users. Continuous intraoperative neuromonitoring (cIONM) allows real-time nerve function during surgery that will reduce the risk of injury. The procedure involved is not without complication thus meticulous usage and strict adherence to standard operating procedure is of utmost importance. This study was aimed to investigate the potential morbidity of the procedure. MATERIALS AND METHODS: A prospective observational study was conducted on 20 patients who underwent routine thyroid surgery using cIONM. The procedure of cIONM adhered to the standard guidelines. Measurements of vagal stimulation were taken proximal (V3) and distal (V2) to the automatic periodic stimulation (APS) electrode to document any injury to the vagus nerve. Blood pressure and pulse rate were also documented throughout the surgery, in order to observe the physiological effect of the stimulation. The function of the vocal cord was assessed by pre and post-operative laryngoscopy. RESULTS: 18 patients with 29 nerves at risk were included as the vagal stimulation did not achieve the acceptable amplitude $(500 \mathrm{~mA})$ in two patients. There were drops of amplitude between V3 and V2 in 14 nerves at risks but there was no clinical evidence of palsy on laryngoscopy. There was also the fluctuation of mean arterial pressure and pulse rate but not significant enough to warrant intervention. CONCLUSION: The use of cIONM during thyroidectomy does exert a minimal effect on the vagus nerve. It is safe and able to help the surgeon identify and preserve the nerve function especially in difficult thyroidectomy.

\section{INTRODUCTION}

Thyroidectomy either in the form of lobectomy, subtotal thyroidectomy, or total thyroidectomy is still one of the most common procedures performed by a general surgeon, head and neck, or endocrine surgeon. Each of the procedures carries a different risk to the recurrent laryngeal which but is very low when performed by an experienced surgeon at a dedicated centre. The introduction of either intermittent or continuous intraoperative nerve monitoring in uncomplicated cases does not show significant improvement in the risk of injury to the nerve as the risk is already very low1 but becomes significant in more complicated cases. 2 The complication of vagal nerve dissection and stimulation during surgery has been documented as needing further investigations ${ }^{3-5}$. Thus, this study aims to investigate the morbidity associated with vagal nerve dissection and stimulation during thyroidectomy.

\section{MATERIAL \& METHOD}

This is a prospective observational study on continuous intraoperative neuromonitoring (cIONM) in 20 patients who underwent thyroid surgery for different diagnoses. The surgery was performed by a single surgeon at an established teaching hospital and included benign and malignant cases. Patients with previous nerve palsy as documented by laryngoscopy was excluded. The cIONM was performed following standard guidelines 
using a commercially available nerve monitoring system, the NIM_ 3.0 Nerve Integrity Monitoring System, and the APS (Automatic periodic stimulation) Electrode by Medtronic, Minneapolis, MN.

The patients were intubated following standard procedure, using an ultra-short acting muscle relaxant on induction. NIM EMG tube by Medtronic was used and the size of the tube was decided by the anaesthetist. No muscle relaxant was given throughout the surgery and the general anaesthesia was maintained either by total intravenous anaesthesia using remifentanil and propofol or inhalational agent isoflurane or sevoflurane.

\section{APS Electrode placement}

The initial step in the placement of the electrode was done through the Lateral Approach. The medial border of the sternocleidomastoid muscle was dissected and retracted laterally. This was followed by dissection of the inferior border of the superior belly of the omohyoid muscle, which was retracted superiorly. This exposes the internal jugular vein (IJV) and the ansacervicalis nerve. Dissection was continued using a scissor to expose the carotid sheath on the medial part of the IJV, lateral to the common carotid artery. Vagus nerve was identified and confirmed with stimulation of $1.0 \mathrm{~mA}$ using the nerve probe (V1). Further dissection of around $1 \mathrm{~cm}$ length of the vagus nerve was made for the application of the APS electrode. A tonsil square or small gauze was inserted to prevent dislodgement of the electrode. The cIONM was observed by applying $1.0 \mathrm{~mA}$ through the APS electrode, set at $1 / \mathrm{s}$. A baseline of more than $400 \mu \mathrm{V}$ was required and manipulation of the tube was made to achieve the targeted level.

Stimulation of the vagus nerve at $1.0 \mathrm{~mA}$ was performed before dissecting the vagus nerve (V1), and after completion of surgery below the point of APS electrode (V2) and above the point (V3) (Figure 1). Demographic data were collected and outcome variables including amplitudes of vagus stimulation (V1V3), laryngoscope findings pre-and post-op, intra-op blood pressure, and pulse rate were documented.

\section{RESULTS}

20 patients were recruited but two patients were excluded because the vagus stimulation did not reach IMJM Volume 20 No.3, July 2021

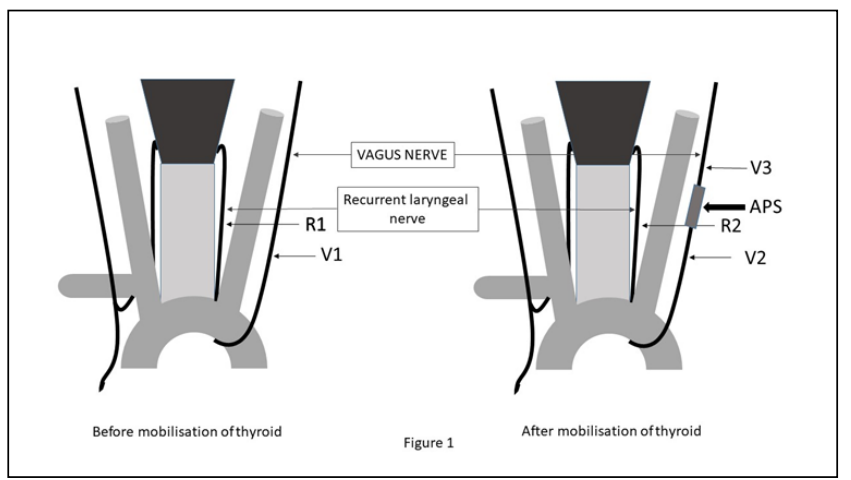

Figure 1. The point of stimulation before and after thyroidectomy.

the acceptable amplitude in all the measurements. In total 18 patients were included with 11 total thyroidectomies and 7 lobectomies, giving rise to 29 nerves at risk. One was excluded due to nondocumentation of V3. Laryngoscopies performed pre and post-operation did not show any evidence of vocal cord palsy. In $14(50 \%)$ nerves at risk, drop in the amplitude between 18 to $217 \mathrm{~mA}$ with an average of $67.7 \mathrm{~mA}$ were noted although no evidence of vocal cord paresis was seen on laryngoscopy. The biggest percentage of drop was $43.5 \%$ although no evidence of vocal cord palsy on laryngoscopy was seen. The remaining nerves did not show a drop in amplitude and even gave higher amplitude than V2 in some of the dissected vagus nerves (Figure 2). Fluctuations of mean arterial pressure throughout the surgery was noted but these were not significant enough to stop the procedure (Figure 3). Similarly, fluctuations of the minimum pulse rate with the biggest drop of 20 beats/minute did not warrant stopping the operation (Figure 4).

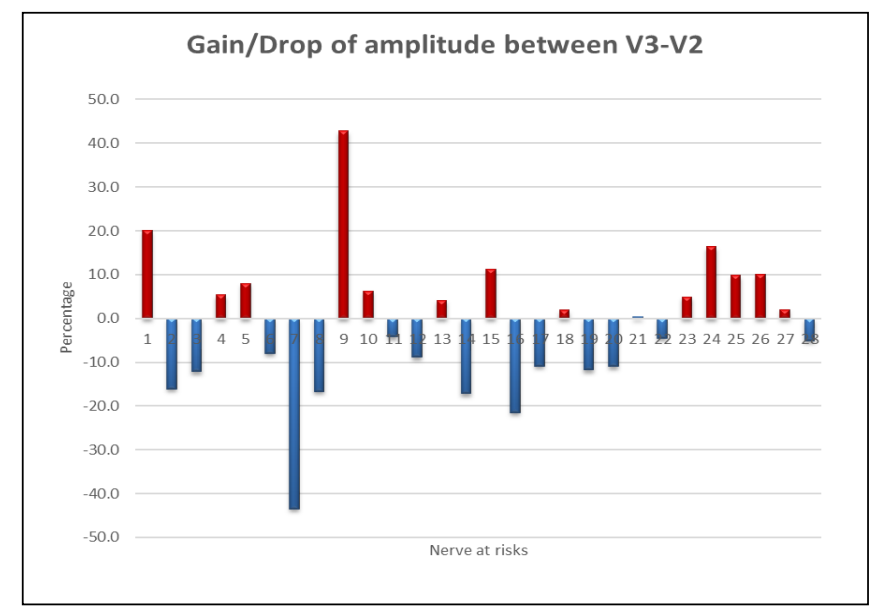

Figure 2. The percentage of amplitude drop/gain following dissection and application of APS at the vagus nerve (V3-V2).

\section{DISCUSSION}

Continuous intraoperative neuromonitoring is becoming more frequent especially in complicated thyroid 


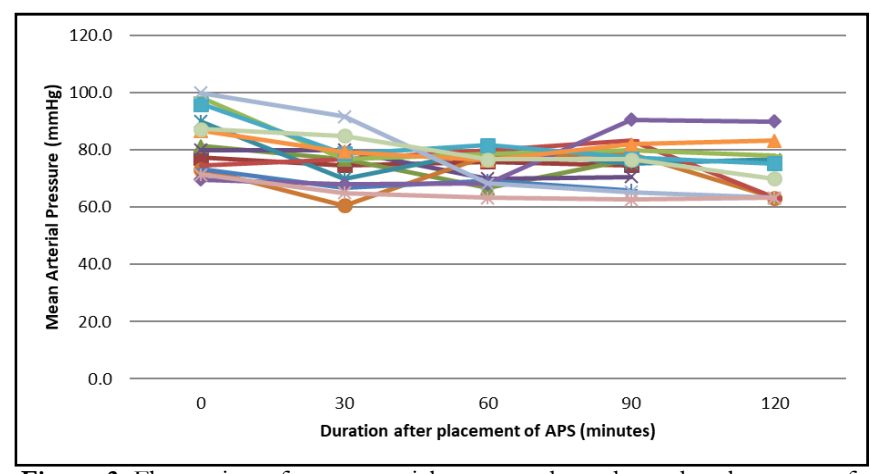

Figure 3. Fluctuation of mean arterial pressure throughout the placement of APS.

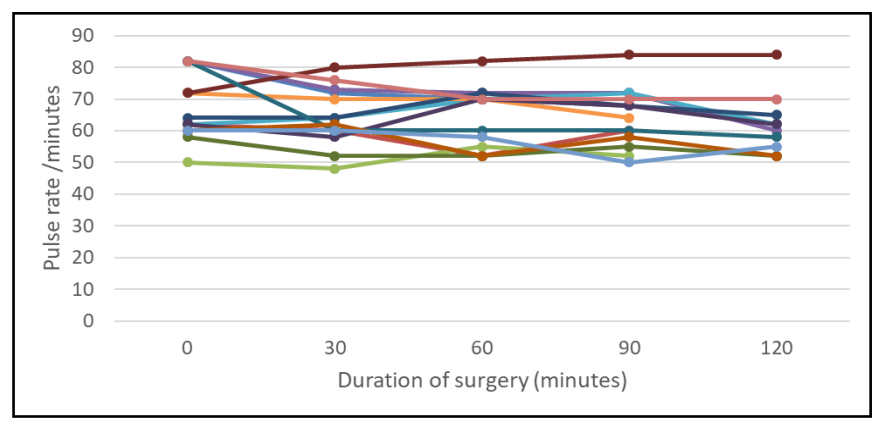

Figure 4. Fluctuation of pulse rate throughout the placement of APS.

surgeries. It uses the principle of automated periodic electrical stimulation of the vagus nerve to allow real monitoring of the recurrent laryngeal nerve function during thyroidectomy. Impending injury will be manifested by an increase in the latency of more than $10 \%$ and also by the drop of amplitude by more than $50 \%$ (double events). ${ }^{6-8}$ The damage becomes irreversible once the amplitude drops to less than $100 \mu \mathrm{V}$ (Loss of signal),. cIONM has the advantage over visual identification or intermittent nerve monitoring as in latter, injury to the nerve could happen in between stimulation or due to stretching of the nerve. ${ }^{9}$ Various large prospective cohort studies have demonstrated the safety of vagal stimulation although there were a few reports on its complications, mainly vagus nerve injury and cardiac complications. ${ }^{3-5}$

Terris et al reported on a case series of 9 patients with 12 nerves at risk where the APS electrodes were dislodged 11 times which might have contributed to the injury to the vagus nerve. ${ }^{4}$ In our study, the lateral approach was used where the carotid sheath was opened after dissecting the anterior border of sternocleidomastoid muscle laterally. By doing so, the APS wire can easily be secured by sticky tape to the lateral end of the wound. A small gauze or tonsil square was used to pack the APS electrode to prevent dislodgement. Only $1 \mathrm{~cm}$ of the vagus nerve was dissected using sharp dissection to reduce ischaemic injury caused by damage to the vasa nervorum. The application of the APS electrode was done meticulously according to the standard practice ${ }^{7}$, preventing traction injury to the vagus nerve.

We measured the amplitude proximal (V3) and distal (V2) to the placement of APS electrode after surgery and found that, in $50 \%$ of the nerve at risk, there was reduction in the amplitude with the highest drop of $43.5 \%$ compared to V2. But this drop in the amplitude did not result in vocal cord palsy. The drop in the amplitude could be contributed by the damage of the nerve during the dissection, placement of the clamp, or due to repeated stimulation of the vagus nerve. Phelan et al demonstrated similar findings in their study, where V2 or post-op vagal stimulation performed proximal to the segment occupied by the electrode after its removal did not show any adverse effects from either vagal dissection or vagal electrode placement in any of their patient. ${ }^{7}$ In the other $50 \%$ of the nerve at risk, the V3 was surprisingly higher than V2. This could be due to improper placement of the nerve stimulator or inadequate dissection of the tissue around the vagus nerve, which provides impedances and reduces conductivity.

Various studies in the past had shown that Automated Periodic Stimulation was not associated with significant adverse neural, cardiac, pulmonary, or gastrointestinal vagal side effects. ${ }^{10-15}$ The strength of stimulation used was $1.0 \mathrm{~mA}$ which was shown in a previous study to not cause any harmful vagal effect. ${ }^{7}$ In our study, the blood pressure and pulse rate were analysed at the interval of 30 minutes to demonstrate the trend of the fluctuation throughout the surgery. Both the mean arterial pressure and pulse rate showed minimal fluctuation during the surgery and no incidence where anaesthetic intervention were recorded.

\section{CONCLUSION}

cIONM is becoming popular among head and neck surgeons to preserve the function of the recurrent laryngeal nerve during thyroidectomy, especially in complicated cases. Stimulation of the vagus nerve at 1.0 $\mathrm{mA}$ is safe and does not cause unwanted physiological effects. Damage during dissection of the vagus nerve depends very much on the technique employed as well 
as meticulous dissection, lateral approach to the nerve, and the use of gauze-pack to prevent accidental dislodgement of the APS clamp.

\section{Conflict of interest}

The study was performed without the involvement of any grant or support by any industry.

\section{REFERENCES}

1. Pisanu A, Porceddu G, Podda M, CoisA, Uccheddu A. Systematic review with meta-analysis of studies comparing intraoperative neuromonitoring of recurrent laryngeal nerves versus visualization alone during thyroidectomy. J Surg Res. 2014;188 (1):152-61

2. Loch-Wilkinson TJ, Stalberg PL, Sidhu SB, Sywak MS, Wilkinson JF, Delbridge LW: Nerve stimulation in thyroid surgery: is it really useful? ANZ J Surg 2007, 77:377-380.

3. Shindo M. Chheda NN: Incidence of vocal cord paralysis with and without recurrent laryngeal nerve monitoring during thyroidectomy. Arch Otolaryngol Head Neck Surg 2007, 133:481-485.

4. Terris DJ, Chaung K, Duke WS. Continuous vagal nerve monitoring is dangerous and should not routinely be done during thyroid surgery. World J Surg. 2015; 39:2471-6.

5. Almquist M, Thier M, Salem F. Cardiac arrest with vagal stimulation during intraoperative nerve monitoring. Head Neck 2016;38: E2419-20.

6. Brauckhoff K, Vik R, Sandvik L, et al. Impact of EMG changes in continuous vagal nerve monitoring in high-risk endocrine neck surgery. World J Surg. 2016; 40:672-80.

7. Phelan E, Schneider R, Lorenz K, et al. Continuous vagal IONM prevents recurrent laryngeal nerve paralysis by revealing initial EMG changes of impending neuropraxic injury: a prospective, multicenter study. Laryngoscope 2014; 124:1498-505.

8. Schneider R, Randolph GW, Sekulla C, et al. Continuous intraoperative vagus nerve stimulation for identification of imminent recurrent laryngeal nerve injury. Head Neck 2013; 35:1591-8.

9. Schneider R, Sekulla C, Machens A, et al. Postoperative vocal fold palsy in patients undergoing thyroid surgery with continuous or intermittent nerve monitoring. Br J Surg. 2015; 102:1380-7.

10. Scott AR, Chong PS, Hartnick CJ, Randolph GW. Spontaneous and evoked laryngeal electromyography of the thyroarytenoid muscles: a canine model for intraoperative recurrent laryngeal nerve monitoring. Ann OtolRhinol Laryngol 2010; 119:54-63.

11. Lamade W, Ulmer C, Seimer A, et al. A new system for continuous recurrent laryngeal nerve monitoring. Minim Invasive Ther Allied Technol 2007; 16:149-154.

12. Schneider R, Przybyl J, Hermann M, Hauss J, Jonas S, Leinung S. A new anchor electrode design for continuous neuromonitoring of the recurrent laryngeal nerve by vagal nerve stimulations. Langenbeck's archives of surgery/Deutsche Gesellschaft fur Chirurgie 2009; 394:903-910.

13. Tomoda C, Hirokawa Y, Uruno T, et al. Sensitivity and specificity of intraoperative recurrent laryngeal nerve stimulation test for predicting vocal cord palsy after thyroid surgery. World J Surg 2006; 30:1230-1233.

14. Lamade W, Meyding-Lamade U, Buchhold C, et al. [First continuous nerve monitoring in thyroid gland surgery]. Chirurg 2000; 71:551-557.

15. Ulmer C, Friedrich C, Kohler A, et al. Impact of continuous intraoperative neuromonitoring on autonomic nervous system during thyroid surgery. Head Neck 2011; 33:976-984. 